\title{
How many frequencies to use in electrical bioimpedance measurements
}

\begin{abstract}
The required frequency range of electrical bioimpedance (EBI) measurements depends on the dielectric properties of the object and covers about three to four decades typically within the $\mathrm{kHz}$ to $\mathrm{MHz}$ range. The accuracy of fitting of the equivalent circuit-based impedance model with the measurement results depends on the number of frequency components used. In general, the use of more frequencies improves the fitting accuracy but complicates the measurements. However, the fitting accuracy also depends on the signal-to-noise ratio (SNR) of measurement signals. If covering the frequency range with a simultaneous multifrequency excitation, the root mean square values of its frequency components are decreasing when their number increases. It follows that the number of used frequency components has two contradictory effects on the accuracy of estimation of parameters of the equivalent circuit model. This chapter deals with the analysis of typical EBI model-fitting situations and shows how the number of frequency components influences the fitting error. Our research shows that in the case of multifrequency excitation the accuracy of fitting of the typical equivalent circuit-based EBI model may not increase when the number of frequencies is increasing. The results described here could be useful in other implementations of impedance spectroscopy.
\end{abstract}

Keywords: electrical bioimpedance, impedance measurement, impedance spectroscopy, equivalent circuit model, estimation of parameters

\section{Introduction}

Electrical bioimpedance (EBI) spectra are widely used to characterize the structure of tissues and cell cultures [1]. For the characterization of biological matter, wide band impedance spectroscopy is required that typically covers three to four frequency decades.

\footnotetext{
Acknowledgement: The work presented in this chapter has been supported by the institutional grant IUT19-11 of the Estonian Research Council and the European Regional Development Fund both supporting the Estonian IT Center of Research Excellence EXCITE and Competence Centre ELIKO.
}

Jaan Ojarand, Raul Land, Mart Min, Thomas Johann Seebeck Department of Electronics, Tallinn University of Technology, Tallinn, Estonia, e-mail: Jaan.Ojarand@ttu.ee

Marek Rist, Thomas Johann Seebeck Department of Electronics, Tallinn University of Technology, Tallinn, Estonia; and Eliko Competence Centre, Tallinn, Estonia 
An important efficiency criterion of the of impedance measurements is the signalto-noise ratio (SNR) which is proportional to the power of measurement signal. In EBI measurements, unfortunately, there is no possibility to improve the SNR by increasing the overall amplitude of the excitation signal since it is limited due to different reasons, such as non-linearity and safety requirements, as well as power consumption and some other technical issues $[2,3]$.

In situations where the properties of the measurement objects are changing in time, e. g., a beating heart muscle or fast moving cells in a high-throughput microfluidic channel, the frequency range of interest must be covered within a short time frame. The use of multifrequency excitations containing several frequency components simultaneously as a multisine signal does [3] gives us a possibility to perform the measurement procedure in a much shorter time span than in the case of subsequent measurements at a single hopping or sweeping frequency. We consider such cases in this chapter. However, the disadvantage is that energy of the multifrequency excitation spreads among multiple frequency components, which decreases the power of each component in the case of a limited total amplitude of the signal.

Another factor, characterizing the power limitation of individual signal components, is the crest factor (CF) of a complex excitation signal - the ratio of its peak and root mean square (RMS) values. In the current analysis, we use a multisine signal (a sum of several sine waves with different frequencies) as the multifrequency excitation. For a graspable comparison of results, some variables must be predetermined. Since the overall amplitude of signals is limited, it is reasonable to normalize the amplitude (peak value $A_{p k}$ ) of the sum of $k$ components of the multisine $\operatorname{Max}|s(t)|=A_{p k}=1$. In the case of equal amplitudes of $k$ multisine components, their individual RMS values are also equal and depend only on a number of components $k$ and the CF [3], i. e.,

$$
S_{R M S}(i)=\frac{A_{p k}}{\sqrt{k} \times C F}=\frac{1}{\sqrt{k} \times C F} .
$$

The units of signal $S$ can be either V or A, depending on the choice to use voltage or current excitation. In Fig. 1, the essentials of formation of the EBI described in [4] and a simplified equivalent circuit of EBI are illustrated.

At lower frequencies, current passes mostly through extracellular fluid. At higher frequencies, when the reactance of the membrane capacitances becomes lower, it also passes through intracellular fluid. A more accurate electrical model also includes both, a constant phase element (CPE) acting at lower frequencies ( $\alpha$ dispersion range) and a stray capacitance of the measurement circuitry. The required frequency range depends on dielectric properties and the structure of the sample under test (SUT) covering decades within the $\mathrm{kHz}$ to $\mathrm{MHz}$ band [1].

The accuracy of fitting of the frequency response curve with the equivalent circuit composed on the bases of measurement results depends on the number of components in the equivalent circuit, the shape of the frequency response of impedance 


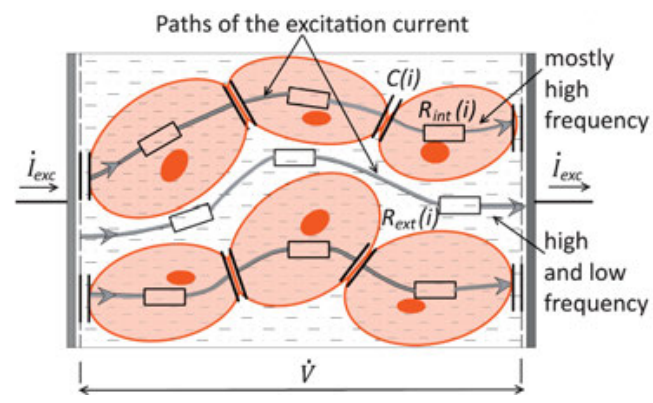

(a)

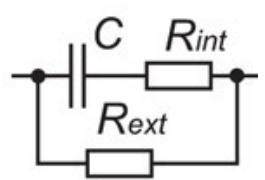

(b)

Fig. 1: (a) Essentials of formation of the EBI. (b) The three-element equivalent circuit.

and, finally, also on the number of frequency components used. The use of more frequencies improves the accuracy because the shapes of the curves of the spectra are followed better. However, the shapes of EBI spectra are relatively smooth since the elements of the equivalent circuit are not producing resonances.

Furthermore, the power of noise and its spectral properties have a substantial negative impact on the fitting accuracy.

Not many results of the systematic study of the number of required frequencies are available. Analysis of the three-element equivalent circuit obtained with sequential single-frequency excitation is described in [5] and [6].

\section{Methodology}

A method used for testing of the influence of the number of frequencies $k$ on the accuracy of estimation of values of equivalent circuit elements is illustrated in Fig. 2. Computer simulation of all the parameters and the equivalent circuit at the predetermined known values of its model elements are used instead of a real SUT. In real experiments, there are more variables, e. g., parameters of electrodes, internal noises of

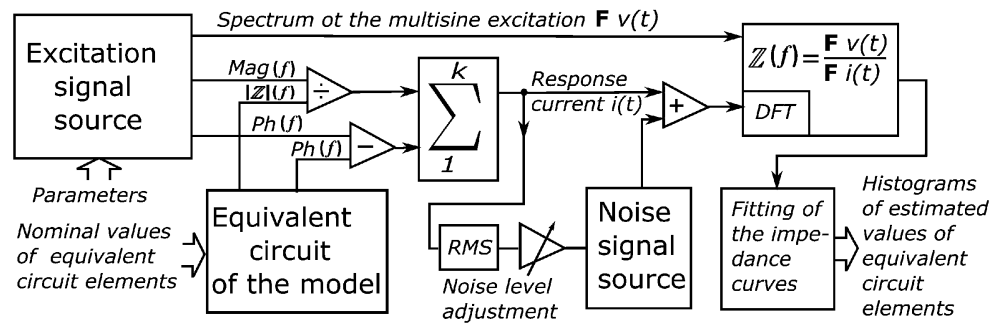

Fig. 2: A simplified structure of calculations used for testing of the influence of the number of frequencies $k$ on the accuracy of the estimation of values of equivalent circuit elements. 
the SUT, stray impedances of the connection wires and measurement circuitry. Moreover, an equivalent circuit model of real SUT is not accurate. The use of synthetic data fixes the number of variables and allows focusing on the dependence of the fitting accuracy on a number of excitation frequencies.

It is assumed that the amplitude of the multisine excitation stays on the level of \pm 1 $\mathrm{V}$, and the initial phases of frequency components are optimal for achieving the best CF. An optimization algorithm described in [3] is used.

We have used five quasi-logarithmically distributed frequency sets in the current analysis. Three decades are covering the frequency range from $1 \mathrm{kHz}$; see Table 1 . The number of frequency points per decade varies from 1 to 5 . Amplitudes of all the sinusoidal components of the excitation voltage are kept equal, but their value is $V(i)=$ $\sqrt{2} \times V_{\mathrm{RMS}}(i)$, the value that decreases when increasing the number of components; see equation (1).

Table 1: Frequency sets of multisines and corresponding crest factors.

\begin{tabular}{rlr}
\hline $\boldsymbol{k}$ & List of frequencies $(\mathbf{k H z})$ & $\mathbf{C F}$ \\
\hline 3 & $1,19,401$ & 2.43 \\
6 & $1,3,13,47,167,607$ & 2.77 \\
9 & $1,3,7,19,41,83,179,379,797$ & 2.58 \\
12 & $1,3,5,7,17,29,47,79,139,241,421,727$ & 2.41 \\
16 & $1,3,5,7,11,17,23,31,47,73,109,163,241,359,523,787$ & 2.22 \\
\hline
\end{tabular}

A simple three-element equivalent circuit model (Fig. 1) and a more complicated sixelement equivalent circuit model (Fig. 3) containing a constant phase element (CPE) were both used.

Magnitudes of each frequency component of the response current were obtained as a ratio of the magnitudes of the excitation voltage and impedance of an equivalent circuit, as shown in Fig. 3. The phase of each component of the response current was found similarly by subtracting the phase values. A waveform of the response current

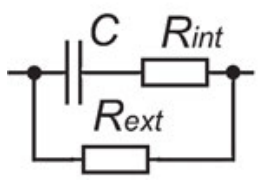

(a)

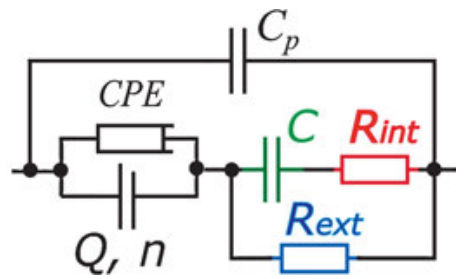

(b)

Fig. 3: (a) Three-element and (b) six-element equivalent circuit models with values of $C=5 \mathrm{nF}$, Rint $=1 \mathrm{k} \Omega$ and $R e x t=560 \Omega$. The additionally introduced elements have values $\mathrm{Cp}=10 \mathrm{pF}, n=0.8$ and $Q=10^{-6} S$. 
$i(t)$, in Fig. 2, is a sum of $k$ sinusoidal components with magnitude and phase values determined by: (a) - known multisine excitation waveform; and (b) - primarily unknown values introduced by the impedance of an equivalent circuit.

Uniformly distributed Gaussian noise adds to the response signal in the next step, and the spectrum of the summary signal is calculated using the Fourier transform (FT) with 4096 points of the waveforms. For a graspable comparison of the results, the RMS value of added noise remains constant when the number of a frequency component varies. The impedance spectrum as the ratio of spectra of excitation and response signals is calculated, and the fitting of noisy impedance curves with a frequency response of the equivalent electrical circuit is performed. Then the differences between the obtained and ideal values are calculated, and the data for histograms are collected.

The fitting algorithm minimizes the mean square error (MSE) as a relative measure of the residuals between the modeled curve values and the actual observed values. $\mathrm{Pa}$ rameters of all components of the equivalent circuit are fitted at once. Along with the residuals also the relative importance of each component of the equivalent circuit at all the frequencies is shown, which allows the user to judge the adequacy of the model and to adjust its parameters if necessary; see the example in Fig. 4. In the current experiments, however, a priori knowledge of the model's topography and the range of parameters simplified the task.

Finally, the errors of mean values and their standard deviations are compared for a different number of frequency components.

The SNR of the response signal is kept at the level of $10 \mathrm{~dB}$, and 500 readings of the response and excitation signal spectra are used for each fitting of model parameters. Then, 500 fitting results are chosen for calculating the standard deviation of the error level for each number of frequencies.

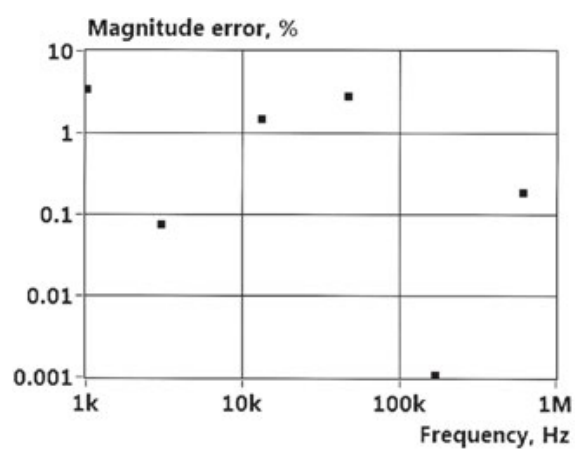

(a)

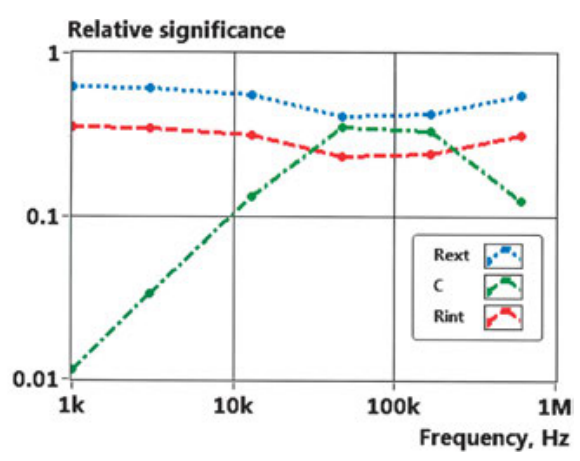

(b)

Fig. 4: (a) A snapshot of the magnitude spectrum fitting error and (b) relative significance of components in formation of the magnitude spectrum, in the case of a three-element equivalent circuit (described in Fig. 3 (a)) with multisine excitation with six components. The upper dotted line in (b) represents Rext, the dashed line represents Rint and the dash-dotted line represents $C$. 


\section{Results}

In Figs. 5 and 6, snapshots of noisy spectra used in experiments for the fitting of values of three-element and six-element equivalent circuits are shown.The SNR of the response signal is kept at the level of $10 \mathrm{~dB}$ in all cases and histograms of the $500 \mathrm{fit}-$ ting results have been collected.

In Fig. 7, histograms are shown of the 500 fitting results collected in the case of the three-element equivalent circuit for different values of $C$ and Rint using multisine excitation with three components.

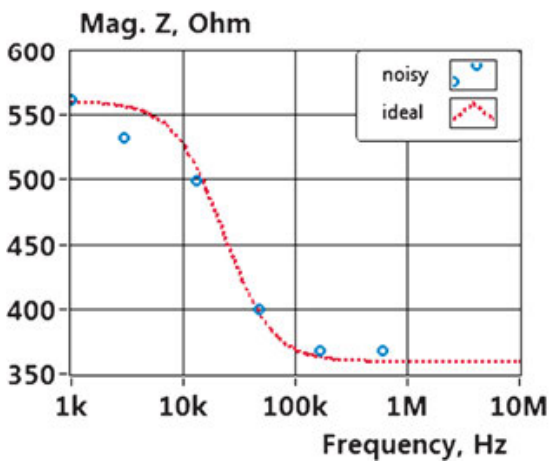

(a)

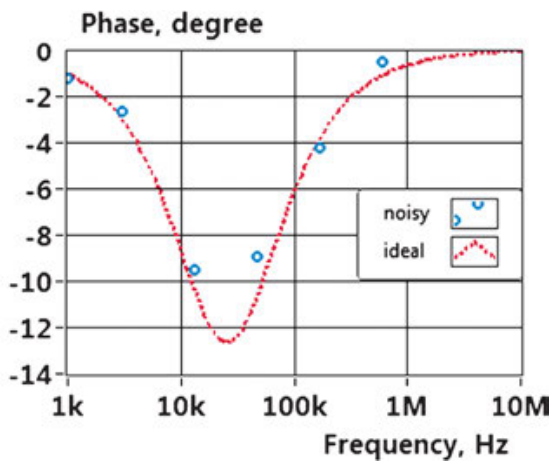

(b)

Fig. 5: (a) Snapshots of the noisy magnitude and (b) phase spectra, in the case of a three-element equivalent circuit (shown in Fig. 3 (a)) and multisine excitation with six frequency components. The dotted lines represent calculated ideal spectra and the circles denote instant values of spectra at used frequencies.

Mag. Z, Ohm

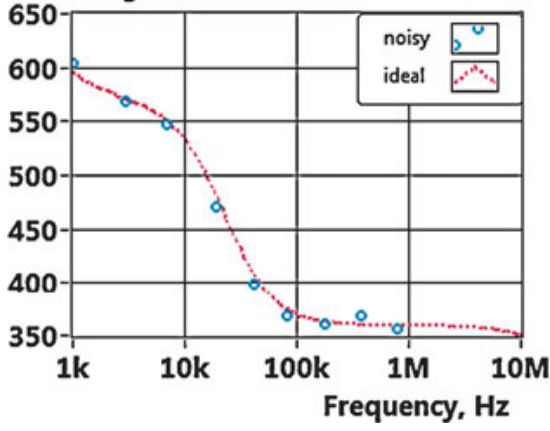

(a)

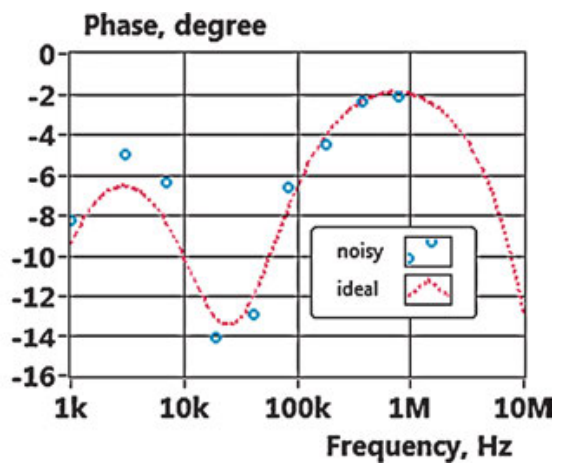

(b)

Fig. 6: (a) Snapshots of the noisy magnitude and (b) phase spectra, in the case of a six-element equivalent circuit (shown in Fig. 3 (b)) and multisine excitation with nine frequency components. The dotted lines represent calculated ideal spectra and the circles denote instant values of spectra at used frequencies. 


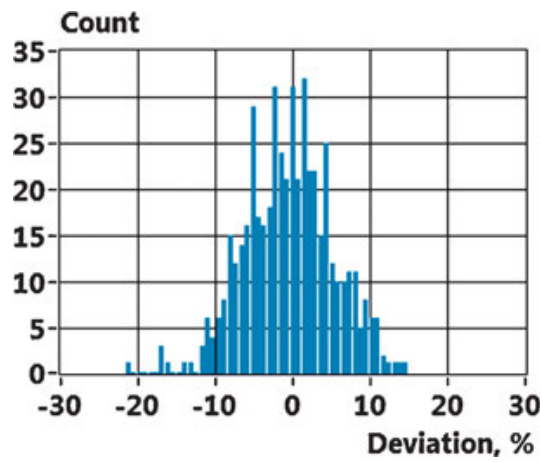

(a)

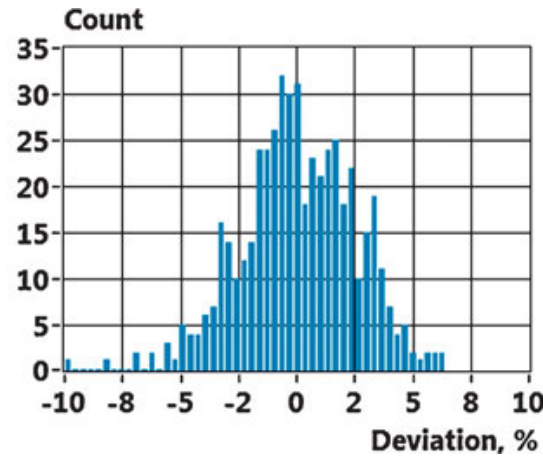

(b)

Fig. 7: Histograms of 500 fitting results of the values of (a) three-element equivalent circuit elements $C$ and (b) Rint given for the multisine excitation having three components.

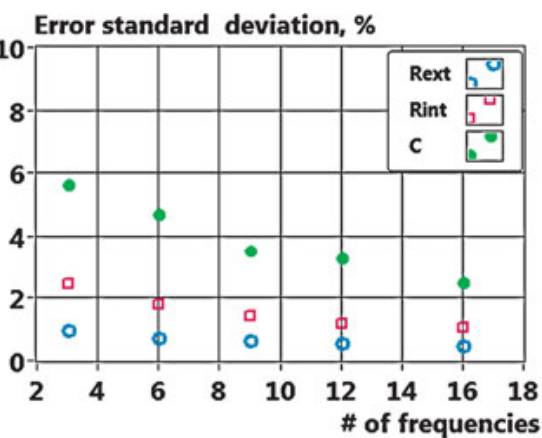

(a)

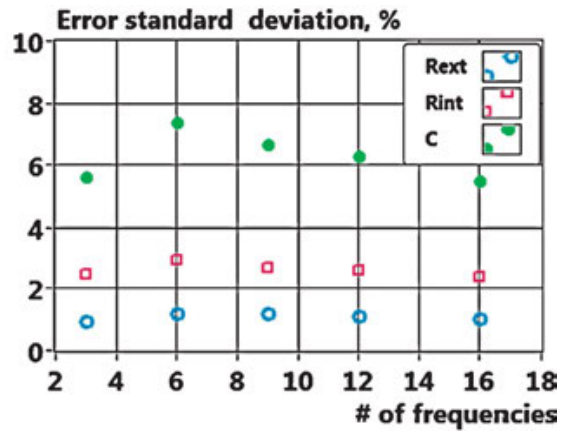

(b)

Fig. 8: The standard deviation of the fitting error of circuit element values of three-element equivalent circuit elements in the case of (a) sequential single frequency excitation and (b) multifrequency excitation; circular dots represent $C$, rectangles represent Rint and circles represent $R e x t$.

The dependence of the standard deviation of the fitting error from the values of threeelement and six-element equivalent circuit elements on the number of frequencies is illustrated correspondingly in Fig. 8 and Fig. 9.

\section{Conclusion}

Increasing the number of frequencies has a low impact on the accuracy of fitting results in the case of using multifrequency excitation and simple three-element equivalent circuits. Even three frequencies could provide sufficiently close results. Reduced energy of individual signal components appearing when adding the frequencies restrains the effect of using a larger number of frequencies. 


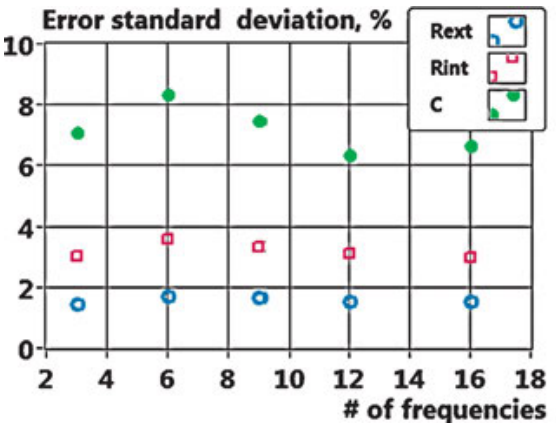

(a)

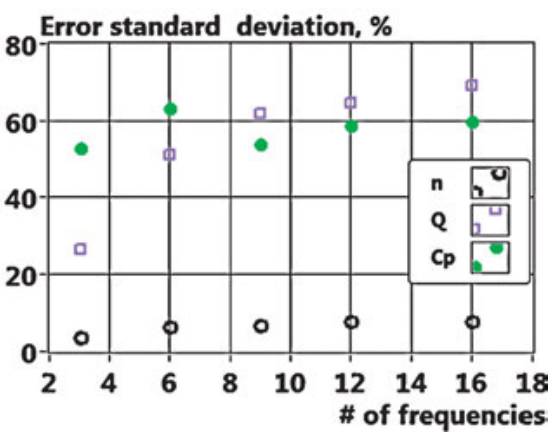

(b)

Fig. 9: Standard deviation of the fitting error of the values of elements of six-element equivalent circuit in the case of (a) multifrequency excitation and (b) a corresponding equivalent circuit. Values of $C$, Rint and Rext are the same as of the three-element circuit in Fig. 1, but the values of additionally introduced elements are $\mathrm{Cp}=10 \mathrm{pF}, n=0.8$, and $Q=10^{-6}$.

It is reasonable to keep the number of frequencies as low as possible at the acceptable errors. Expectedly, the number of frequencies should be larger, if the noise distributes non-uniformly over the frequencies or some concentrated disturbances appear at some frequency sub-ranges. Every specific case deserves special handling.

The positive effect from the use of more frequencies is larger in the highly noisy environment if the single-frequency signals are applied sequentially for excitation, but then the measurement process becomes too long for the measurements of timevarying impedances.

\section{Bibliography}

[1] S. Grimnes and Ø. G. Martinsen, "Bioimpedance and Bio-electricity Basics”, 3rd ed., Elsevier-Academic Press, pp. 77-403, 2015.

[2] U. Pliquett, “Electricity and biology”, In: Proc. BEC2008, Tallinn, Estonia, pp. 11-20, 2008.

[3] J. Ojarand and M. Min, "Crest factor optimization of the multisine waveform for bioimpedance spectroscopy”, Physiological Measurement, vol. 35, pp. 1019-1033, 2014.

[4] M. Min, T. Parve, and U. Pliquett, “Impedance detection”, In: Encyclopedia of Microfluidics and Nanofluidics, 2nd ed., (Ed.) Prof. Dongqing Li, Springer, Berlin-Heidelberg, 28 pp., 2015.

[5] L. Ward and B. Cornish, "Multiple frequency bioelectrical impedance analysis, how many frequencies to use ?”, In: Proc. ICEBI XII and EIT V, vol. 61, pp. 321-324, 2004.

[6] S. Kun and R. A. Peura, "Selection of measurement frequencies for optimal extraction of tissue impedance model parameters”, Medical \& Biological Engineering \& Computing, vol. 37, pp. 699-703, 1999. 